\title{
Syllable cut and energy contour in vowels: a comparative study on German and Hungarian
}

\author{
Katalin Mády \\ Pázmány Péter Katolikus Egyetem, Germanisztikai Intézet, Piliscsaba, Hungary
}

Krisztián Z. Tronka

Pázmány`Péter Katolikus Egyetem, Germanisztikai Intézet, Piliscsaba, Hungary

\author{
Uwe D. Reichel \\ Institut für Phonetik und Sprachliche Kommunikation, Ludwig-Maximilians- \\ Universität München, Germany
}

Syllable cut is said to be a phonologically distinctive feature in some languages where the difference in vowel quantity is accompanied by a difference in vowel quality like in German. There have been several attempts to find the corresponding phonetic correlates for syllable cut, from which the energy measurements of vowels by Spiekermann (2000) proved appropriate for explaining the difference between long, i.e. smoothly, and short, i.e. abruptly cut, vowels: in smoothly cut vowels, a larger number of peaks was counted in the energy contour which were located further back than in abruptly cut segments, and the overall energy was more constant throughout the entire nucleus. On this basis, we intended to compare German as a syllable cut language and Hungarian where the feature was not expected to be relevant. However, the phonetic correlates of syllable cut found in this study do not entirely confirm Spiekermann's results. It seems that the energy features of vowels are more strongly connected to their duration than to their quality.

\section{Introduction}

The German vowel system is characterised by a correlation of vowel quantity and vowel quality: long vowels are normally tense, while short vowels are lax, cf. [ii] - [I]: Miete 'rent'-Mitte 'centre', [e:] - [ع]: Weg 'way' - weg 'away' etc. 
It has been an object to discussion for decades whether one of both features is predictable from the other and can therefore be regarded as redundant.

One group of phonologists treats the quantity as the primary phonological (or even the only phonologically relevant) feature in this opposition. However, quantity is an accent-phenomenon in German, i.e. long vowels occur mainly in stressed position. An appropriate description must thus assume a set of rules shortening an underlying long vowel in an unstressed syllable in order to provide the correct surface forms, cf. Musik [u] ' '[i:] 'music' - Musiker '[u:] [I] 'musician' - musikalisch [U] [I] '[a:] 'musical' - Musikalität [U] [I] [a] [I] '[e: $]^{2}$ 'musicality'. Other phonologists propose that the distinctive feature is rather tenseness. Since this feature remains intact in the alternation above, such an analysis can describe it in a more plausible way without assuming rules changing an underlying feature in the surface representation. However, the assumption of distinctive tenseness is in one respect unsatisfactory: there are several connections between the vowel opposition and prosodic phenomena (quantity, stress, phonotactic equivalence between long vowels, diphthongs and short vowel + consonant combinations etc.) - indicating that this opposition is probably not a segmental one.

Another solution of the problem is based on the assumption of a syllable cut opposition in Standard German. The basic idea of this concept is that stressed short lax vowels are somehow "not perfect" in the sense that they require a postvocalic segment in the same syllable, while short (if unstressed) or long (if stressed) tense vowels do not. The described problems of the other two concurring theories are avoided in this concept since (1) the opposition of abrupt cut (scharfer Schnitt) with a lax vowel and smooth cut (sanfter Schnitt) with a tense vowel is clearly a prosodic one and (2) temporal differences between the two vowel classes are just concomitant phonetic phenomena (or even side effects) of this higher suprasegmental contrast. Despite of its phonological plausibility, this concept was often rejected in the second half of the $20^{\text {th }}$ century - because of the lacking phonetic correlate of the syllable cut in Contemporary German.

In his study, Spiekermann (2000) discussed and investigated all phonetic correlates for vowel segments that had been assumed so far by phonologists from Sievers through Trubetzkoy up to Vennemann and Maas \& Tophinke (for references, see Spiekermann, 2000). Spiekermann found that the parameters used to describe energy contours were highly relevant for the contrast abrupt vs.

1 While prescriptive transcriptions suggest that a short tense $[\mathrm{u}]$ is pronounced here, most natives would prefer [U].

2 According to Northern Standard German and everyday speech. In elaborated speech, the last vowel is pronounced as [E:] instead of [e:]. 
smooth cut: 'E-Max' (Germ E-Zahl, number of energy peaks), 'E-Pos' (position of the energy maximum) and 'E-Hold' (Germ E-Halt, difference between energy minimum and maximum divided by the maximum). According to Spiekermann's results, smoothly cut (i.e. tense and long) vowels had more energy peaks that were located further back in the segment, and smoothly cut vowels had a higher intensity level throughout the entire segment than abruptly cut vowels. The tendency for the energy maximum to be located further back in smoothly cut and earlier in abruptly cut vowels lead Spiekermann to the assumption that the main characteristics of the syllable are not to be found in the nucleus-coda transition as proposed by Sievers, but in the onset-nucleus transition.

Spiekermann also tested vowel oppositions in Finnish and Czech that primarily make use of a quantitative opposition and thus are not regarded as syllable cut languages. He found that in all languages, longer durations are associated with a higher E-Max, while E-Pos and E-Hold were more or less indifferent for duration. These values were either located between smooth and abrupt cut in German or were closer to the measures for abrupt cut.

While Spiekermann's results are impressing, there are two main shortcomings in the experimental setup. Firstly, he relied on a relatively small corpus $(n=225)$ that involved all VC combinations of German uttered only once, thus, no statistic analysis could be undertaken. Secondly, his analysis was carried out manually, and the parameters were expressed in three categories instead of metric (i.e. percent) values.

There are strong phonological arguments for the assumption that syllable cut is not crucial for the Hungarian vowel system (Tronka, 2005). First, while a German syllable including a short vowel is only well formed if the vowel is followed by a consonant, short vowels can be syllable final (i.e. they do not require a coda) in Hungarian (eg. falu /a/, /u/, 'village'). Second, the relevance of syllable cut was primarily restricted to accented syllables, as it is the only position where vowel quantity is distinctive in German (and most Germanic languages). In Hungarian, however, vowel quantity is independent of word stress (which is always on the first syllable in the word), c.f. falat /'falat/ 'mouthful' - falát /'fala:t/ 'his/her wall'.

Like German, Hungarian involves seven vowel classes, (/i, y, u, e, ø, o, a/), of which all can be realised long or short (Mády, 2001). The main vowel opposition in Hungarian is durational, while long and short /e/ and /a/ also differ in quality. There is a smaller quality opposition in $/ 0 /$ and $/ \varnothing /$, where the laxness of the short vowel is mostly explained by dynamic effects, and which most speakers of Hungarian are not aware of (Siptár \& Törkenczy, 2000).

Based on the assumption that syllable cut plays a central role for German vowels but it is not relevant for Hungarian, it was hypothesised that the features 
E-Max, E-Norm, E-Pos, and E-Hold were relevant for the distinction between long (smoothly cut) and short (abruptly cut) vowels in German. At the same time, long and short vowels were not expected to differ significantly for Hungarian along their energy patterns, but to behave similar to Finnish and Czech.

In order to test Spiekermann's finding for Hungarian, we constructed a pilot study (Tronka, Mády \& Reichel, 2006) with slightly modified parameters based on metric instead of ordinal scales (see 2.2). Our results for German were not completely comparable with those in Spiekermann (2000): while smoothly cut vowels included more energy maxima which were located further back in the segment, their overall energy showed a greater minimum-maximum difference than that of abruptly cut vowels. Moreover, exactly the same tendencies were found for Hungarian where syllable cut was not supposed to apply.

However, the German and Hungarian corpora available at that time were not entirely comparable (little overlap of consonant environment), thus we felt it necessary to perform the analysis on a more appropriate speech material. The measures will first be tested for German and Hungarian separately. On the basis of these findings, the results from the two languages will be compared and discussed.

\section{$2 \quad$ Material and methods}

\subsection{Material}

Both the German (4 speakers) and the Hungarian ( 3 speakers) corpora ${ }^{3}$ included /i, y, u, e, ø, o, a/ as short and long vowels in nonsense words embedded in a carrier sentence (including 6 syllables in the German corpus and 9 in the Hungarian one). German words had the structure $/ \mathrm{C}_{1} \mathrm{VC}_{2} \mathrm{a} /$ where $\mathrm{C}_{1}$ and $\mathrm{C}_{2}$ were stops and had the same place of articulation ( $\mathrm{PoA}$ ) (labial or velar), while $\mathrm{C}_{1}$ was voiced and $\mathrm{C}_{2}$ unvoiced. The structure of the Hungarian stimuli was slightly different: the last vowel was /a/ plus $C_{1}$ and $C_{2}$ were identical. Consonants were varied for PoA (labial, alveolar and velar). Both corpora were balanced for vowel duration and quality and consonant PoA $[\mathrm{n}($ germ $)=1076$, $\mathrm{n}$ (hung) $=1006$.

The speech material was segmented automatically (by the software MAUS from the Department of Phonetics and Speech Communication in Munich) and corrected manually (in Praat 4.3). F2 onset and offset were applied as a boundary marker. As shown in Figure 1, some vowels offer two

3 Both corpora have been recorded at ZAS, Berlin, for articulatory investigations by Electromagnetic.Midsagittal Articulography (EMMA). 
interpretations of the segment boundary: (1) the first and last appearance of F2 in the segment (including transitions), (2) the first and last appearance of the entire formant structure including F3 and F4 and thus including only the central, relatively steady phase of the vowel. Our preference of the first alternative relies on the assumption, that syllable cut is primarily based on certain requirements of syllable structure and not just on the vowel, thus, juncture (before and after the nucleus) will probably play a central role in its physical manifestation. Thus, it seems convincing to concentrate on the entire vowel duration.

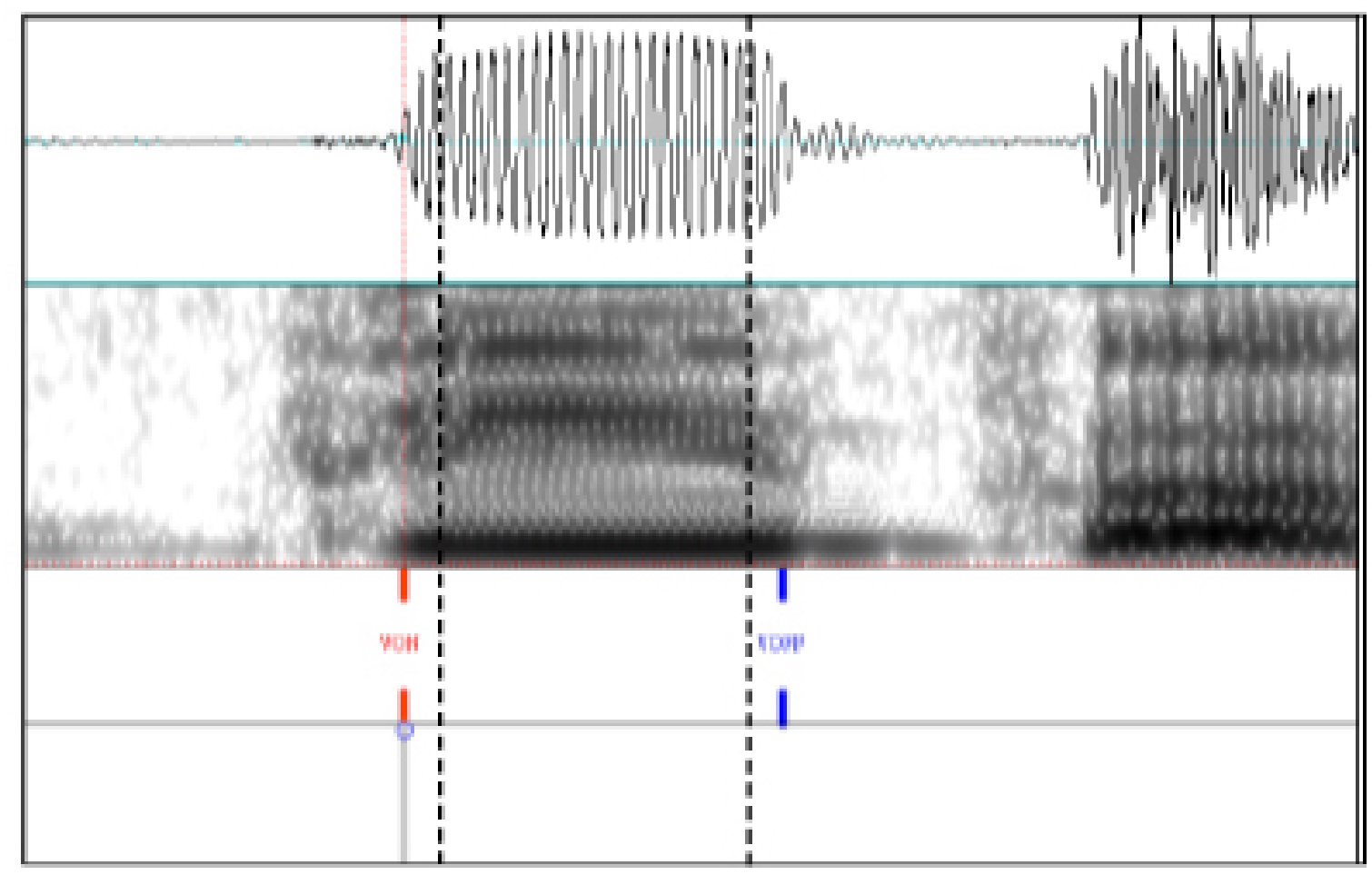

Figure 1: Segmentation technique shown by the example of the Hungarian item /pipp/. (1) Left and right segment boundary: onset and offset of F2, (2) dotted lines between them: onset and offset of entire formant structure (including F3 and F4).

\subsection{Methods}

Smoothed energy contours were calculated by applying overlapping Hanning windows of $20 \mathrm{~ms}$ on the rectified oscillogram in order to remove glottal closure peaks.

Following measures, based on Spiekermann (2000) but not in full accordance with this study, were then derived from these contours (see also Figure 2): 
- E-Max: the absolute number of maxima,

- E-Norm: E-Max normalised to the length of the contour (number of peaks divided by number of samples),

- E-Pos: the relative position of the last maximum within the contour,

- E-Hold: the ratio of the difference between the absolute maximum and minimum with respect to the maximum.

In contrast to Spiekermann we did not only calculate E-Max in absolute terms, because a positive relation between contour length and the number of maxima within this contour is somewhat self-explaining, and as vowels in smoothly cut syllables tend to be longer than in abruptly cut ones, the former will trivially show more energy peaks than the latter.

In order to cancel out this durational effect, we divided the energy peak number by the length of the energy contour. Furthermore, we avoided the loss of information due to data quantification Spiekermann carried out for E-Pos, for which he divided the vowel into 9 segments, and for the quotient E-Hold which had been categorised in 3 different classes. Instead of categorising E-Pos, we directly calculated the relative position of the last maximum with respect to vowel length, and also for E-Hold no classification was done. Therefore in our study the features E-Pos and E-Hold are not ordinally but metrically scaled.

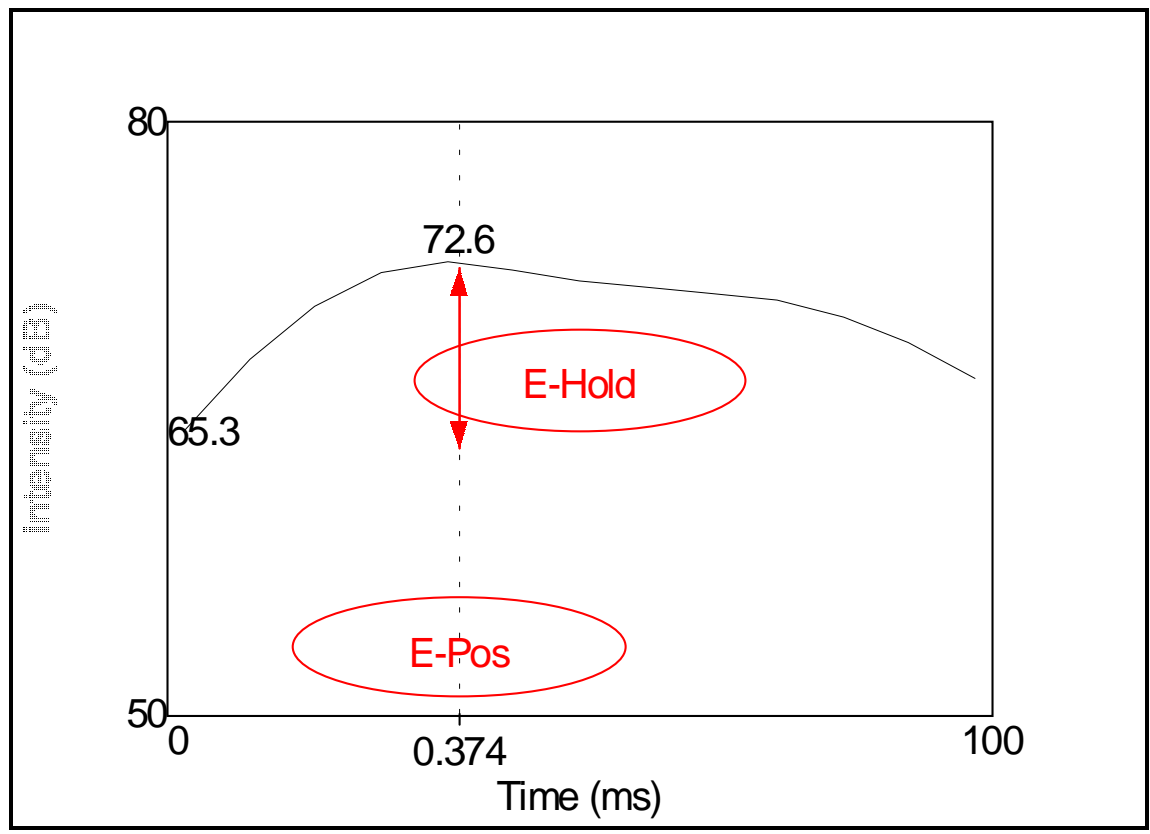

Figure 2: Energy contour of a vowel segment with the parameters E-Max $=1, \mathrm{E}-\mathrm{Norm}=0.0007, \mathrm{E}-\mathrm{Pos}=0.374$, and $\mathrm{E}-$ Hold $=0.095$, duration $=100 \mathrm{~ms}$. 
The measure E-Pos was also modified: in Spiekermann's analysis, only vowels with exactly one energy maximum were included in the analysis. However, if the distinctive character of syllable cut is based on the state of the energy level in the moment when the vowel is cut by the following consonant, then the position of the last energy maximum is relevant, no matter how many peaks were counted before. Thus, we calculated E-Pos as the position of the last maximum, but for reasons of compatibility, E-Pos was also calculated for vowels with one maximum.

\section{Results}

\subsection{German vowels}

\subsubsection{Vowel length}

German long ('l', smoothly cut) and short ('s', abruptly cut) vowels differed significantly for duration with slight overlap of the peripheral values. Short vowels tend to have more outliers (more than 1.5 interquartile distances but less than 3) towards long vowels than the other way around. In other words, while there is a relative contrast between long and short vowels, a given duration value cannot be directly associated with smooth or abrupt cut, and abruptly cut vowels seem to be less attached to a short duration than smoothly cut vowels to a longer one.

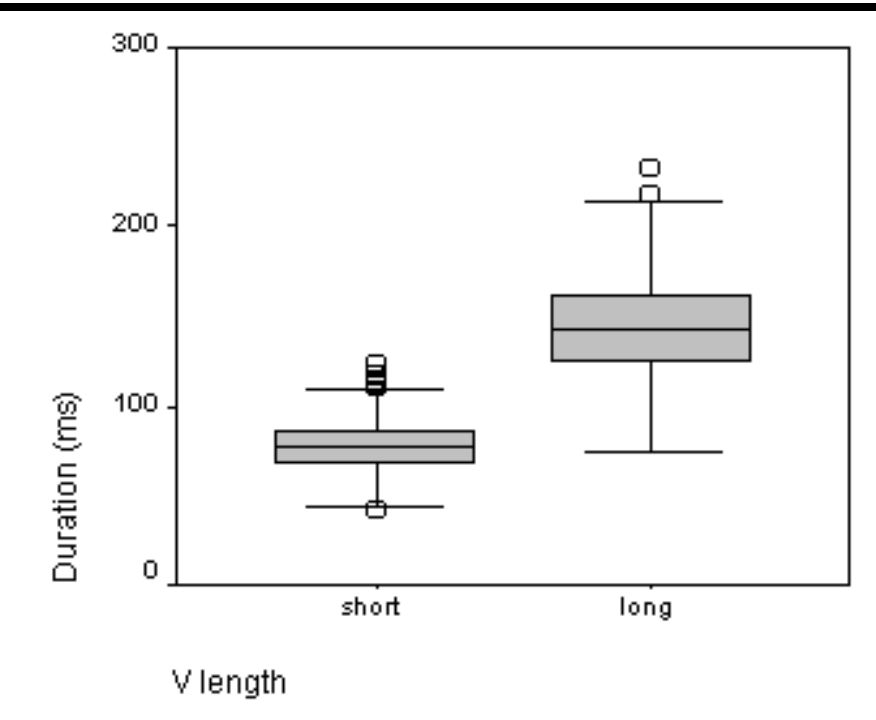

Figure 3: Duration for short (s) and long (l) German vowels. Mean $(\mathrm{s})=78 \mathrm{~ms}, \mathrm{SD}(\mathrm{s})=13 \mathrm{~ms}$, mean $(\mathrm{l})=$ $144 \mathrm{~ms}, \mathrm{SD}(1)=27 \mathrm{~ms}$. 
The parameters duration, E-Max, E-Norm, E-Pos and E-Hold were tested for correlation. None of the energy measures showed a linear correlation. Therefore, Spearman's Rho was calculated over all parameters. No strong correlation (higher than $\rho=0.6$ ) was found between any of the parameters. Duration was correlated positively with E-Max, E-Pos and E-Hold, but negatively with E-Norm, i.e. longer vowels had relatively fewer energy peaks than short ones.

The significance of duration and the energy measures was tested by a $\mathrm{t}$ test for two independent samples ( $\alpha \leq 0.05$, two-tailed). Most data units did not meet the condition of a normal distribution for an ANOVA, but they were large enough to perform a Welch test $(\mathrm{n}>50)$ that does not require normally distributed and homogenous samples.

The difference for all tested variables between smoothly and abruptly cut vowels was highly significant. Long vowels had more energy peaks (E-Max). However, the relative number of energy maxima (E-Norm) was smaller for longer vowels, i.e. they were less dense in long vowels than in short ones (Figure 4a,b). The last energy maximum (E-Pos) was located further back in the vowel segment, as was also found in Spiekermann (2000).

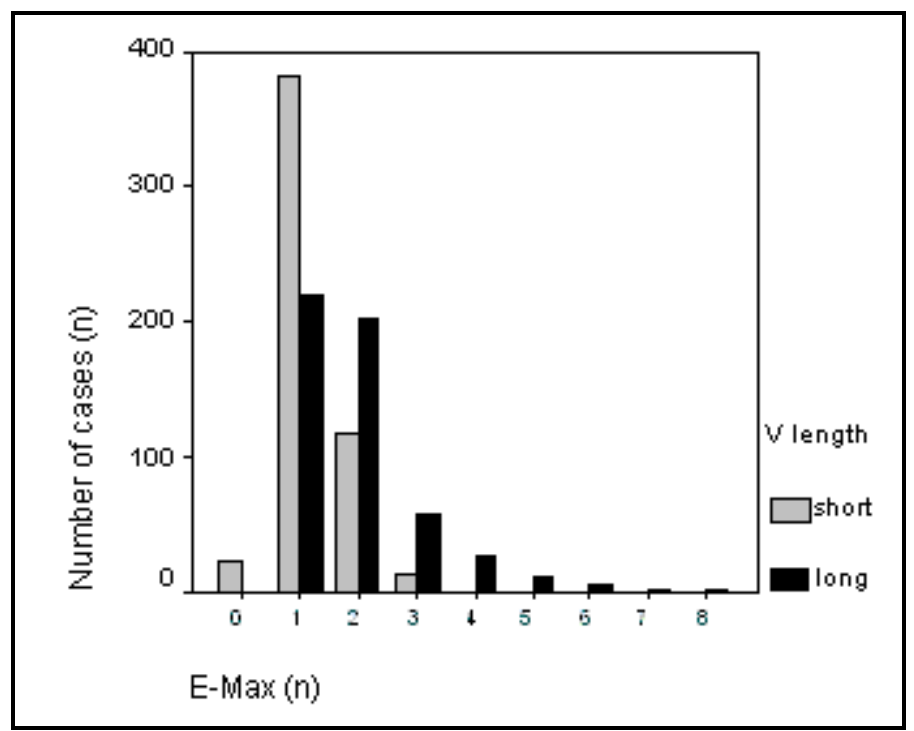



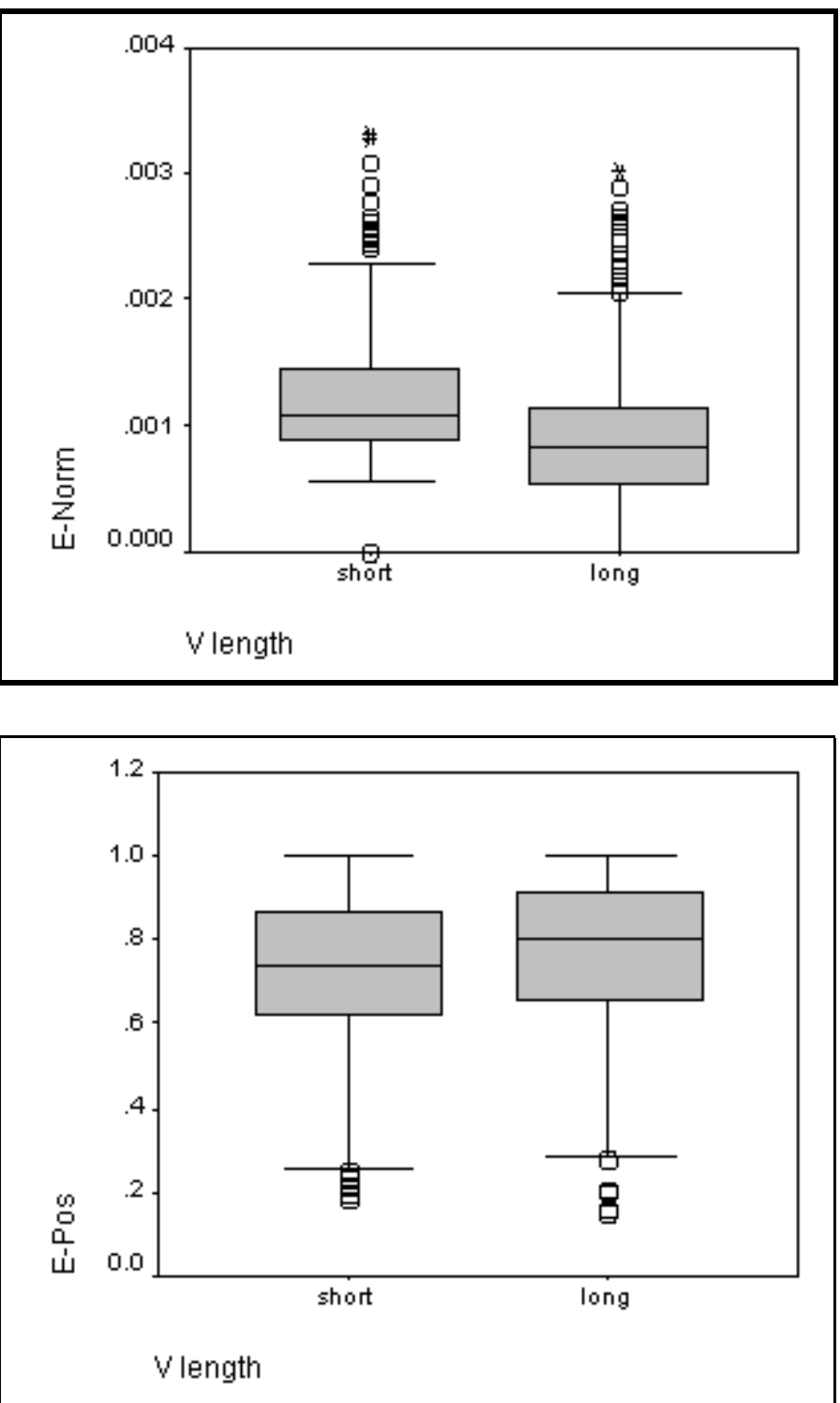

Figure 4a-c: (a) E-Max, (b) E-Norm, (c) E-Pos of short and long German vowels. Box plots: black line: median, upper and lower box, upper and lower whisker: $25 \%$ of cases (interquartiles), respectively.

However, we obtained results different from those of Spiekermann (2000) regarding the overall energy level in the segment. While he found a high intensity level in long vowels throughout the entire vowel segment (E-Hold), our results show exactly the opposite pattern: according to the t-test, the difference between intensity maximum and minimum in long vowels is significantly larger than in short ones. While in Spiekermann's study, long vowels often had an EHold of less than 5\% (0.05 in the present scaling). As shown in Figure 5, such a 
small difference was not found in any segment in our data, the smallest E-Hold being $0.055^{4}$

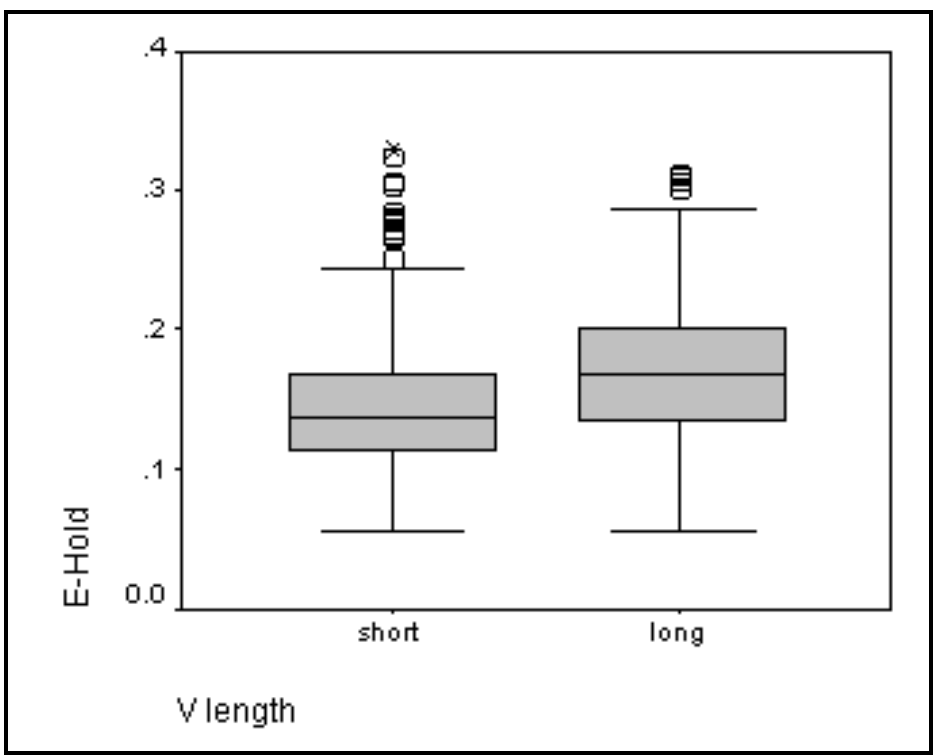

Figure 5: E-Hold of short and long vowels in German.

\subsubsection{Vowel classes}

Duration for each vowel quality (long vs. short) was significantly different (Figure 6). The fact that high vowels are the shortest and low vowels the longest segments corresponds to general tendencies regarding intrinsic duration: high vowels tend to be shorter than low ones in most languages (Kassai, 1998).

4 This might be a consequence of different segmentation guidelines from those used in our corpora. If not the onset and offset of F2, but the entire visible formant structure was used as boundary markers of the vowel, the segment duration is probably shorter and thus, differences within this domain are smaller (see 2.1 and Figure 1). 


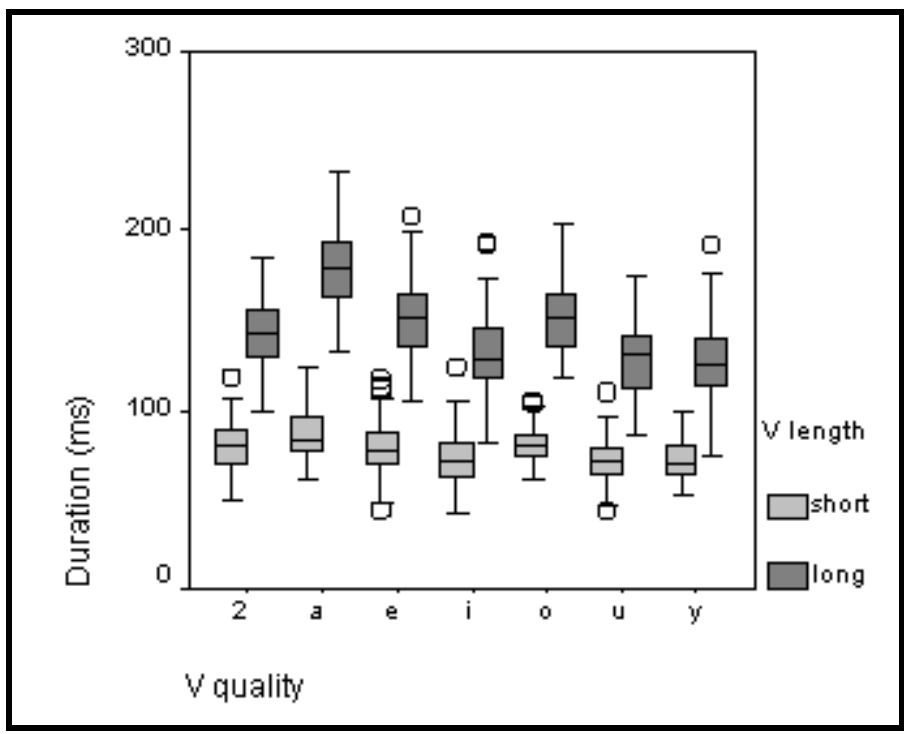

Figure 6: Vowel durations in each German vowel class (vowel quality is given in SAMPA).

In order to test the energy parameters along vowel quality, a t-test was performed for each vowel pair (long and short, approximately 80 realisations per each). While the examined parameter differed in almost all vowel pairs according the pattern described above, the difference was not significant at the $5 \%$ level for any of the vowels except for $/ \varnothing /$.

All long vowels had a larger number of energy maxima. Most vowels (except for $/ \mathrm{a} /$ and $/ \mathrm{u} /$ ) had a higher value for E-Norm.

The least reliable parameter was E-Pos. Three vowels did not show a difference at all $(/ \mathrm{i} /, / \mathrm{o} /, / \mathrm{u} /)$, and in $/ \mathrm{e} /$, the difference was not significant. The tendency in E-Hold was not much clearer: 4 vowels matched the overall pattern, while three (/i, y, a/) did not (Figure 7). If E-Pos and E-Hold were calculated according to Spiekermann's method, the pattern was even less clear. No interaction with vowel height can be seen along the parameters. 

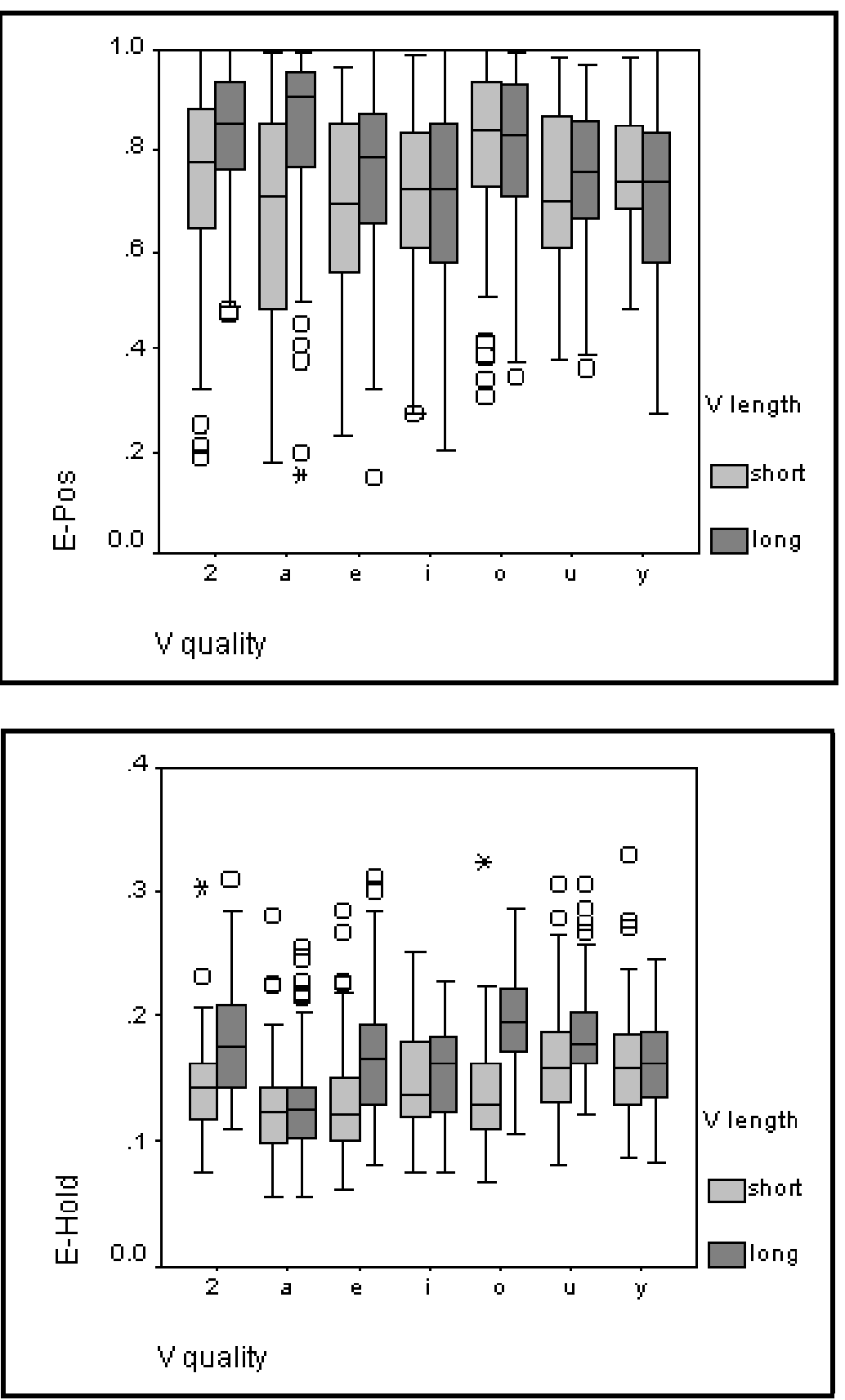

Figure 7a,b: E-Pos and E-Hold in short and long vowels for short and long German vowels (vowel quality is given in SAMPA). Circles stand for outliers (1.5-3 box lengths, i.e. interquartile distances from the upper/lower end of box), asterisks for extreme values ( $>3$ interquartile distances). 


\subsection{Hungarian vowels}

\subsubsection{Vowel length}

Hungarian long and short vowels differed significantly for duration. While Hungarian long vowels were somewhat shorter than German ones, the standard deviation is the same, while short vowels have the same mean but a clearly larger standard deviation. In other words, the difference between short and long vowels was less clear-cut than in German.

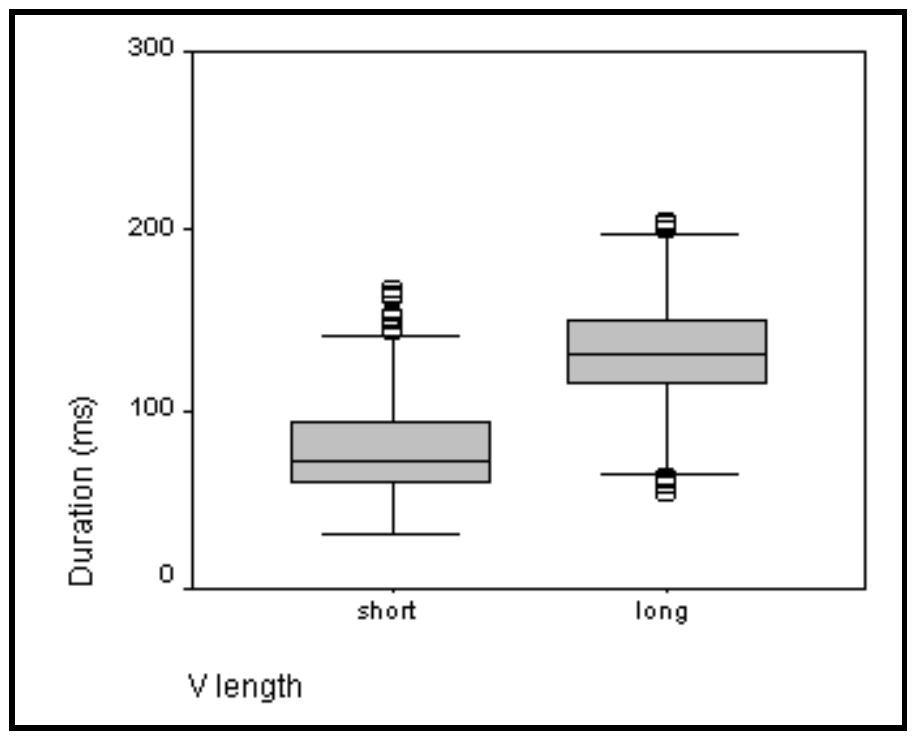

Figure 8: Duration for short (s) and long (1) Hungarian vowels. Mean $(\mathrm{s})=78 \mathrm{~ms}, \mathrm{SD}(\mathrm{s})=24 \mathrm{~ms}$, mean $(\mathrm{l})=$ $132 \mathrm{~ms}, \mathrm{SD}(1)=27 \mathrm{~ms}$.

Correlations between duration and the energy parameters were approximately identical with those in German.

A comparison revealed the same tendencies between long and short vowels regarding E-Max and E-Norm: a higher number of energy peaks in long vowels, but their higher density in short vowels. E-Pos was located further back in long vowels that also had a larger maximum-minimum difference of intensity. E-Norm and E-Hold resembled the pattern described for German.

\subsubsection{Vowel classes}

All vowel classes differed significantly for duration. Short vowels showed, as seen in Figures 6 and 9, little variation of duration. The difference between the long vowel and its short counterpart was largest in /a/ and smallest in $/ y /$. This finding is interesting in the context that long and short /a/ in Hungarian clearly 
differ for quality ([a: $]$ vs. $\left.[\mathrm{p}]^{5}\right)$, while high vowels do only to a small extent (Kassai 1998). Figure 9 reveals a large overlap between short and long /y/. It is interesting, as Vicsi \& Szaszák found that the shortest vowel in their corpus (BABEL) was the long vowel /y:/. It seems, that the duration distinction plays only a marginal role for this sound. ${ }^{6}$

All vowel classes match the results found for the entire set of data, but none of the classes reveals a significant difference for all three parameters.

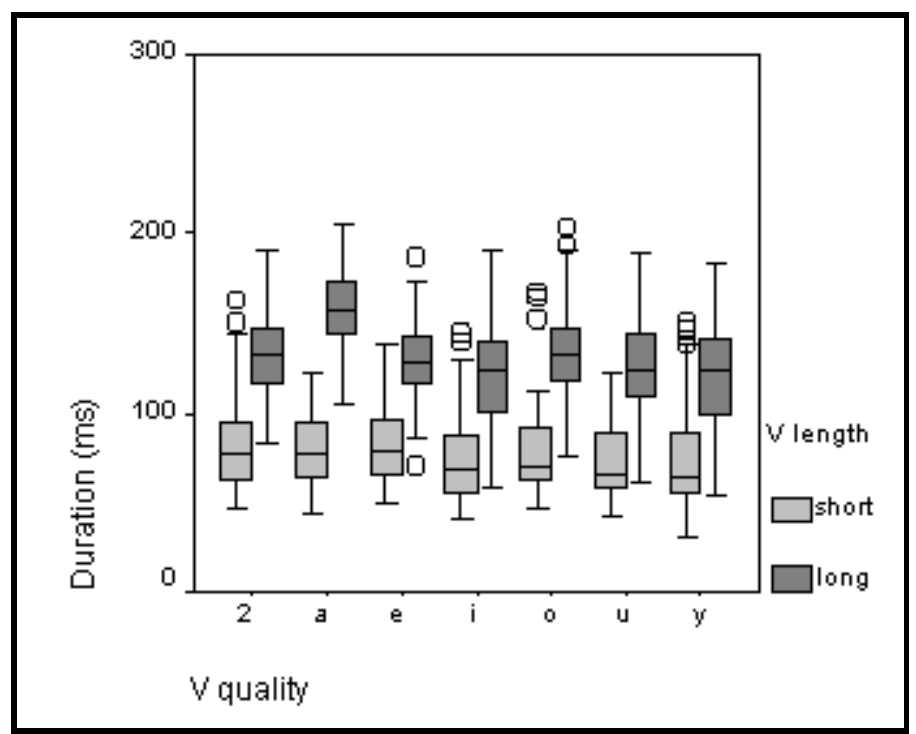

Figure 9: Vowel durations in each Hungarian vowel class (vowel quality is given in SAMPA).

\subsection{Contrasting German and Hungarian vowels}

As said in 2.1, both the German and the Hungarian corpora involved slightly different consonant contexts. Although both consonant PoA and voicing might have an impact on vowel duration, this could be ignored in the previous sections because each corpus was balanced for these factors. However, a comparison between German and Hungarian vowels required a corpus where consonantal environment was identical for both languages. Therefore, only stimuli with labial and velar consonants were considered for the contrastive corpus $(\mathrm{n}=1747)$.

5 Short /a/ is normally given as [0] in Hungarian phonetics, but the vowel quality is better expressed by the 13. cardinal vowel [p].

6 These findings are unpublished and rely on personal communication with György Szaszák, Department of Telecommunication and Media Informatics, Budapest University of Technology and Economics. 


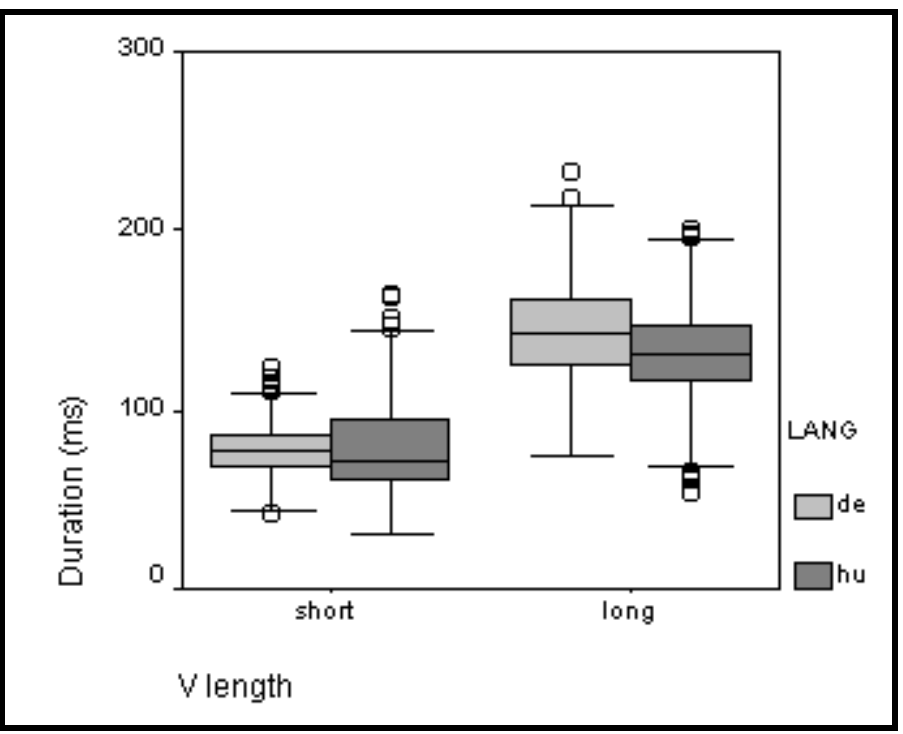

Figure 10: Duration for short and long German (de) and Hungarian (hu) vowels. Mean $(\mathrm{Germ})=144 \mathrm{~ms}$, $\mathrm{SD}($ Germ $)=27 \mathrm{~ms}$, mean $($ Hung $)=131 \mathrm{~ms}, \mathrm{SD}($ Hung $)=$ $28 \mathrm{~ms}$.

As shown in Figure 10, long vowels in German and Hungarian differ significantly for duration. Therefore the energy parameters were again compared with regard to German and Hungarian long vowels.

The whole set of long German and Hungarian vowels differed significantly for E-Norm, and E-Pos, but not for E-Hold. In short vowels (that were not significantly shorter in Hungarian) the same tendency was observed. The energy parameters were related to duration in the same way as for each language: German vowels, that were significantly longer than Hungarian ones (see Figure 10), had a higher number of absolute but a lower number of relative maxima, which were located further back in a vowel segment, and the overall energy level was lower.

Finally, German and Hungarian vowels were compared class-wise. While the German vowels were longer in each case, the difference was not significant for the high vowels $/ \mathrm{i} /, / \mathrm{y} /$, and $/ \mathrm{u} /$. These vowels had shorter durations in both languages than mid-high and low vowels, and the duration was less specific in Hungarian (expressed by a high SD, especially for /y/, see Figure 11).

German vowels, that were longer than their Hungarian counterparts, had significantly lower E-Norm and higher E-Pos values, but there was no clear tendency regarding E-Hold. While the German vowels were expected to have higher E-Hold, i.e. a larger intensity range within the segment, it was only true in four cases: for the middle vowels $/ \mathrm{e} /, / \varnothing /, / \mathrm{o} /$, and for the high back $/ \mathrm{u} /$. 


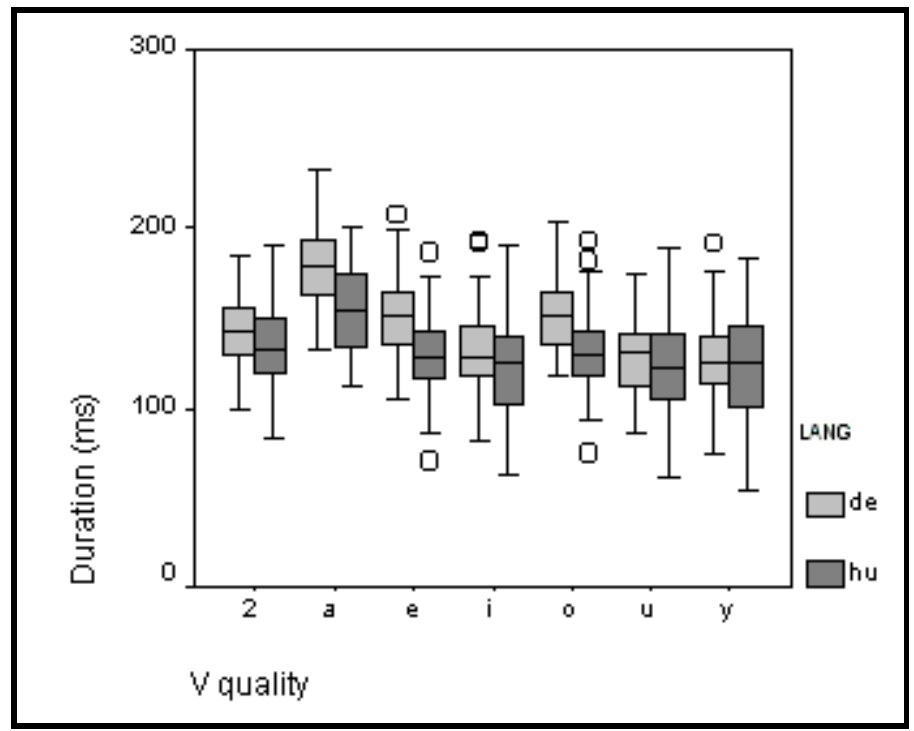

Figure 11: Duration for long German and Hungarian vowels for each of the seven vowel classes.

\section{Discussion in a phonological framework}

The theory of syllable cut is based on languages in which (1) long vowels are associated with tenseness and (2) stressed short vowels always require a consonantal syllable coda. In these languages, vowel length is only distinctive in stressed syllables. The main idea is that smoothly cut (long and tense) and abruptly cut (short and lax) vowels do not only differ in length and tenseness, but there is a hidden, more general category that governs the other two features. According to Spiekermann (2000), this feature can be detected in the different energy contours of the vowels.

After several decades of unsuccessful search for reliable acoustic correlates of the hypothetical syllable cut phenomenon, Spiekermann (2000) proposed that the difference relied on the different energy contours in vowels with smooth and abrupt syllable cut. He also proposed that non-syllable-cut languages like Finnish and Czech did not show the regularities that were found for German. The same tendency was expected for Hungarian which is a nonsyllable-cut language (for arguments, see 1).

In the present study, duration and energy parameters proposed by Spiekermann were re-tested on a larger German and Hungarian speech corpus. The results of the investigation of vowel duration showed a significant difference between long and short vowels for both languages - as was expected. The investigation of the separate vowel classes revealed that German short vowels varied less than the Hungarian ones, while the variation of short and long vowels in Hungarian overlapped in many cases. In one case $(/ y /)$, the short vowel was often realised with a duration that reached beyond the longest 
articulation of its long equivalent. The relative constancy of the German short vowels confirms Trubetzkoy's theory, according to which smoothly cut vowels are expandable while abruptly cut ones are not (see also Hoole \& Mooshammer, 2002, for articulatory data). Becker (1998) sets up a theoretical framework for this phenomenon and suggests that if a syllable in a smoothly cut vowel is expanded, it is always the vowel that is lengthened, while in a syllable with an abruptly cut vowel it is rather the following consonant. The fact that German short and long vowels do not or only slightly overlap shows the stability of the opposition in question: there seems to be a strong tendency for the double distinction (in duration and quality), as syllable cut is not only theoretically distinctive in German, but there are lots of cases (minimal pairs) in which it does in fact differentiate between meanings. In other words, this distinction is very often used in German.

On the other hand, there is no clear duration distinction in Hungarian for some of the vowel classes. Thus, we may assume that the phonological opposition that is manifested phonetically by means of durational differences is not a stable one: an overlap of the short segment far into the central part of its long counterpart signalises that it is not so important to make a clear-cut difference between a short and long vowel. This finding matches well the investigations of Kassai (1979) who pointed out that for some of the vowel pairs of Hungarian, only few minimal pairs exist, thus, she argued that the quantity opposition in this language was relatively instable.

Although our results regarding duration were in accordance with those of Spiekermann (2000), the energy contours we found were often slightly different. In our data, long vowels had more energy maxima than short ones, just as Spiekermann describes. On the other hand, if the number of the maxima was normalised by vowel duration, long (smoothly cut) German vowels had in fact less energy maxima than short vowels, and the energy contours diverged more than in short (abruptly cut) vowels. In other words, while the number of energy maxima in long vowels varied from one to ten, short vowels included three energy peaks at most. Thus, the idea that tense vowels are characterised by a constant and high energy level did not prove to be appropriate. On the contrary, short (lax) vowels seem to have a more compact energy distribution (relatively more energy maxima and a smaller decrease of the intensity level during the vowel segment) according to their higher E-Norm and lower E-Hold values.

These findings are somewhat surprising, as they do not fit the tentative descriptions given by some phonologists (i.e. Becker), who see a direct relationship between syllable cut, the amount of energy, and phonetic manifestation (i.e. duration and tenseness/centralisation of a vowel). They argue that in the case of abrupt cut, there is always a postvocalic segment (typically a 
consonant) cutting the ballistic production of the preceding vowel - the result is a smaller energy content resulting in a short and lax, i.e. more centralised articulation of the vowel in question. In case of smooth cut, there is no postvocalic segment at all or if any, it does not cut the vowel before its energy climax, thus, the vowel is articulated long and tense, i.e. not centralised. On the other hand, the idea of syllable cut could be maintained despite our findings, if one would consider it from the other 'side', i.e. from the perspective of the expandibility concept. In this case, one could argue as follows: in case of a lacking cutting effect (i.e. if there is an unrelated or no postvocalic segment at all in the syllable) a vowel will be expanded, and expansion of a vowel can lead to a nonlinear increase of the number of intensity maxima (i.e. the number of maxima is not directly related to the duration difference), while it is not possible for abruptly cut vowels. This has already been stated regarding speech rate in Hoole \& Mooshammer (2002) from an articulatory point of view. On the other hand, all speculations on the relationship between expandibility, energy content and syllable cut will be superfluous if one considers our results regarding Hungarian, where similar data were found for E-Norm dividing long and short vowels, although no difference was hypothesised on the basis of the syllable cut theory. Hence, the only possible conclusion is that the absolute and relative number of energy maxima in a vowel is rather related to differences in duration than to syllable cut.

As already said previously, one of the main ideas of syllable cut is that abruptly cut vowels are cut by the following segment while smoothly cut segments are not. Spiekermann's (2000) results regarding E-Pos were therefore somewhat unexpected, as he found exactly the opposite tendency as had been supposed by phoneticians and phonologists since Eduard Sievers: the intensity maximum appeared further back in smoothly cut vowels than in abruptly cut ones. At the same time, he found similar patterns in non-syllable cut languages - thus, the only possible interpretation was that also E-Pos is related to duration and not to syllable cut. Our measurements could confirm Spiekermann's data in one aspect: the energy maximum lied further back in long vowels than in short ones, both in German and in Hungarian. On the other hand, we could measure only small differences for E-Pos in both languages (approximately $6 \%$ of the vowel duration), therefore it is questionable whether E-Pos has any phonological relevance at all.

One of the fundamental findings in Spiekermann's investigations are undoubtedly his results for E-Hold. Since he found a relationship between syllable cut and the difference of energy minimum and maximum in a vowel, but he could not find it in non-syllable cut languages, he concluded that E-Hold was to be seen as a stable acoustic correlate of syllable cut in German. Our measurements in German confirmed Spiekermann's results: there are indeed 
differences in the energy range between smoothly and abruptly cut vowels. On the other hand, our results for Hungarian do not support this conclusion, as we found similar data also for Hungarian, where syllable cut does not play a role. Thus, we conclude that also E-Hold is probably rather related to duration than to syllable cut, as it is also present in Hungarian and between German and Hungarian vowels. The situation is more confusing, if one considers the seven vowel pairs in the two languages. A comparison of some German vowel pairs does show a relation between language type and intensity differences found by Spiekermann (mid-high vowels and $/ \mathrm{u} /$ ). So we cannot exclude the possibility in general that E-Hold plays any role in distinguishing between the two language type.

Finally, we want to report about some problematic points of Spiekermann's parameters. It is not quite clear what E-Hold as a central category in Spiekermann's theory really means. We are probably not wrong in the assumption that E-Hold is a kind of acoustic implementation of what Maas (1999) metaphorically called "austrudeln" ('to fade out'). The fact that smoothly cut vowels "fade out" while abruptly cut ones do not, is interpreted such, that smoothly cut vowels have a constant energy contour, while abruptly cut ones show an abrupt change. Spiekermann intends to apprehend this by measuring the difference between the minimum and the maximum value of the energy contour in a vowel. However, there are at least two crucial problems connected to this analysis method. Spiekermann measured - as we did when retesting his hypotheses - an intensity minimum regardless of its position in the global energy contour of the vowel, i.e. regardless of the fact whether it is at the beginning or at the end of the contour. We think that it is probably not unimportant to reduce the investigation only to a certain part of the intensity contour. If we speak about the virtual cutting effect of a postvocalic segment, this points to the $\mathrm{VC}$ transition. If one on the other hand takes the findings for E-Pos into account, one could argue for measuring the CV transition of the energy contour of the vowel in question. There are tentative arguments against the latter approach that are based on accidental findings. The German data we originally intended to use for our analysis had a small echo, so we set the boundary mark before the echo was visible in the oscillogram and the spectogram. This lead to a general shortening of all German vowel segments with the conceivable effect that a possible difference in E-Hold disappeared. As the shortening always took place at the end of the vowel, what might have lead to a neutralisation of the duration difference concerning E-Hold, we assume that acoustic cues of syllable cut are probably rather to be found at the $\mathrm{VC}$ boundary.

Besides, E-Hold is a problematic parameter from the acoustic and perceptual point of view. Due to the nonlinearity of the decibel scale, the 
quotient (energy maximum - energy minimum)/energy maximum still depends on the respective energy level and this lack of normalisation prohibits a comparison of E-Holds derived from different energy levels. Furthermore, EHold does not reflect perceived energy differences, that is, an E-Hold value of 0.1 does not refer to the same perceptive difference if the energy maximum is located at 80 or at $65 \mathrm{~dB}$ in the given segment.

For a better understanding of the phenomenon of syllable cut, several further aspects should be taken into account: what pattern can be found in (1) unstressed vowels, (2) diphthongs, and (3) reduced vowels? Do unstressed vowels that are long and tense phonologically but short in their phonetic realisation like /e/ in Metall show the characteristics of smooth or abrupt cut? Is duration a crucial factor for reduced vowels that are not marked for tenseness? How would the tenseness difference of Hungarian mid-high vowels influence the energy parameters in a larger corpus? The answers to these question may bring us closer to the question whether syllable cut is a relevant feature in the German vowel system.

\section{Acknowledgements}

We would like to thank Christian Geng, Centre for General Linguistics,

Typology and Universals Research (ZAS), Berlin, for providing us with the German and Hungarian data.

\section{References}

Becker, T. (1998) Das Vokalsystem der deutschen Standardsprache. Frankfurt: Lang.

Hoole, P. \& Mooshammer, C. (2002) Articulatory analysis of the German vowel system. In: P. Auer, P. Gilles \& H. Spiekermann (eds.) Silbenschnitt und Tonakzente. Tübingen: Niemeyer, 129-152.

Kassai, I. (1979) Időtartam és kvantitás a magyar nyelvben. Budapest: Akadémiai Kiadó.

Kassai, I. (1998) Fonetika. Budapest: Nemzeti Tankönyvkiadó.

Maas, U. (1999) Phonologie. Upladen: Westdeutscher Verlag.

Mády, K. (2001) Kontrastive Phonetik Deutsch-Ungarisch in Hinblick auf zu erwartende Interferenzphänomene. Linguistische Beiträge Pasmaniensis, 1: 29-51.

Siptár, P. \& Törkenczy, M. (2000) The Phonology of Hungarian. Oxford: University Press.

Spiekermann, H. (2000) Silbenschnitt in deutschen Dialekten. Tübingen: Niemeyer.

Tronka, K. (2005) Die Vokallänge im Deutschen und Ungarischen. In: I. Szigeti (ed.) Junge Germanisten stellen sich vor. Frankfurt: Lang, 177-196. 
Tronka, K., Mády, K. \& Reichel, U. D. (2006): A contrastive study of syllable cut in German and Hungarian: vowel length and energy. $3^{\text {rd }}$ Old World Phonology Conference, Budapest, 17-19. January 2006, 57-58. 